\title{
VAMP/synaptobrevin as an acrosomal marker for human sperm
}

\author{
João Ramalho-Santos, Ph.D., ${ }^{\mathrm{a}}$ Yukihiro Terada, M.D., Ph.D., ${ }^{\mathrm{b}}$ and \\ Gerald Schatten, Ph.D. ${ }^{\mathrm{C}}$ \\ Division of Reproductive Sciences, Oregon Regional Primate Research Center, Oregon Health Sciences \\ University, Beaverton, Oregon
}

Received April 3, 2001; revised and accepted July 23, 2001.

This work was supported by grants from the NICHD/ $\mathrm{NIH}$ to G.S., through cooperative agreement [U54 18185], as part of the Specialized Cooperative Centers Program in Reproductive Medicine; and by a post-doctoral fellowship to J.R.-S. (FCT, Portugal).

Reprint requests: João Ramalho-Santos, Ph.D., Department of Zoology, University of Coimbra, Largo Marquês de Pombal, 3004-517 Coimbra, Portugal (FAX: + $351(239)$ 826798; E-mail: jramalho@ci.uc.pt).

${ }^{a}$ Center for Neuroscience and Cell Biology of Coimbra, Department of Zoology, University of Coimbra, Coimbra, Portugal.

${ }^{\mathrm{b}}$ Department of Obstetrics and Gynecology, Tohoku University School of Medicine, Miyagi, Japan.

${ }^{c}$ Pittsburgh Development Center, Magee-Women's Research Institute, University of Pittsburgh, Pittsburgh, Pennsylvania.

0015-0282/02/\$22.00 PII S0015-0282(01)02957-0

Objective: To determine the possible use of the mammalian acrosomal marker vehicle-associated membrane protein (VAMP)/synaptobrevin to detect acrosome abnormalities in human sperm.

Design: Analysis of human sperm after fixation and staining with an anti-VAMP antibody.

Setting: An academic research institution.

Patient(s): Semen samples from consenting patients who were participating in an infertility treatment program.

Intervention(s): Human sperm samples were fixed, permeabilized with detergent, and examined by immunocytochemistry.

Main outcome measure: Immunostaining.

Result(s): Observation of sperm from patients with no obvious sperm morphological defects revealed normal looking acrosomes, as assessed by VAMP immunostaining. However, severe acrosome malformations were detected in other cases. The observations registered varied from the absence of a fully formed organelle in samples of patients with globozoospermia to abnormal VAMP staining in samples from patients with acrosomal defects.

Conclusion: VAMP/synaptobrevin may be a useful marker for the functional assessment of acrosomal status in human sperm. (Fertil Steril ${ }^{\circledR}$ 2002;77:159-61. ( 2002 by American Society for Reproductive Medicine.)

Key Words: Sperm, acrosome, VAMP/synaptobrevin

The acrosome is a large secretory vesicle localized on the heads of a spermatozoa in most species. Fusion between the acrosome membrane and the sperm plasma membrane (i.e., the acrosome reaction) results in the release of acrosomal contents, thus priming the sperm for egg interactions. Although many aspects related to the triggering of the acrosome reaction have been characterized, little is known on what modulates membrane merging itself.

One protein that has been recently identified on the human acrosome is the vesicle-associated membrane protein (VAMP), also known as synaptobrevin (1). Together with other acrosomal components, VAMP may have an important functional role in promoting membrane fusion during the acrosome reaction (1).

We have previously used VAMP staining to detect the acrosome after intracytoplasmic sperm injection (ICSI), a technique used to overcome certain types of male factor infertility and that allows for the direct injection of a spermatozoon into an oocyte (2). Using the rhesus monkey as a model system, we have shown that a VAMP "collar" (probably corresponding to the acrosome equatorial segment) sharply separates the decondensed (posterior) portion of the sperm head from the apical part, which remains largely condensed (2).

In this study, we have attempted to use VAMP to detect acrosome abnormalities in semen samples from patients who were participating in an infertility treatment program. Although the original clinical diagnosis varied, infertility in all samples was diagnosed as male factor using a recently described assay (3).

\section{MATERIALS AND METHODS}

Semen samples were obtained from consenting infertility patients treated at the Tohoku 
Sperm parameters of patients with male factor infertility analyzed for VAMP acrosomal staining.

\begin{tabular}{|c|c|c|c|c|}
\hline Patient & $\begin{array}{c}\text { Sperm count } \\
\left(10^{6} \mathrm{sperm} / \mathrm{mL}\right)\end{array}$ & $\begin{array}{c}\text { Sperm motility } \\
(\%)\end{array}$ & $\begin{array}{l}\text { Predominant } \\
\text { defect }\end{array}$ & $\begin{array}{l}\text { Original clinical } \\
\text { diagnosis }\end{array}$ \\
\hline 1 & 256.0 & 25.0 & Cells in ejaculate & Male factor \\
\hline 2 & 70.0 & 54.2 & Cells in ejaculate & Tubal \\
\hline 3 & 216.0 & 47.0 & $\begin{array}{c}\text { Lasso tails } \\
\text { Twin tails/heads }\end{array}$ & Idiopathic \\
\hline 4 & 213.0 & 33.0 & $\begin{array}{l}\text { Lasso tails } \\
\text { Twin tails }\end{array}$ & $\begin{array}{l}\text { Tubal and } \\
\text { male factor }\end{array}$ \\
\hline 5 & 18.3 & 20.0 & Globozoospermia & Idiopathic \\
\hline 6 & 184.0 & 73.6 & Globozoospermia & Male factor \\
\hline
\end{tabular}

Note: Patients 1-6 correspond to patients 6, 9, 13, 16, 1, and 17 discussed in Sutovsky et al. (3), respectively.

Ramalho-Santos. VAMP in human sperm. Fertil Steril 2002.

University Hospital (3). All samples were collected and frozen at the Tohoku University Hospital, Japan. All samples were collected and frozen at the Tohoku University Hospital before analysis at the Oregon Health Sciences University. We were unable to analyze fresh samples during the course of this work and therefore cannot assess the possible effects of freezing on VAMP staining.

Samples were used in accordance with the guidelines approved by the Institutional Review Boards (IRBs) of both Tohoku University and Oregon Health Sciences University. Appropriate IRB approval was obtained in both institutions.

Frozen samples were thawed in warm modified Tyrodelactate medium with pyruvate and albumin, and immunocytochemistry using VAMP antibodies (a kind gift of Dr. Gary Wessel, Brown University; and obtained commercially via Synaptic Systems, Göttingen, Germany) was carried out as described in detail elsewhere (1). Briefly, poly-L-lysine coated coverslips with attached sperm were fixed in $2 \%$ formaldehyde, permeabilized in $1 \%$ Triton X-100, and blocked by further incubation in phosphate-buffered saline (PBS) containing $2 \mathrm{mg} / \mathrm{ml}$ bovine serum albumin and 100 $\mathrm{mM}$ glycine. For labeling, anti-VAMP antibody was solubilized in the blocking solution (1:100 dilution) and incubated with the coverslips for $1-2 \mathrm{~h}$ at room temperature. After washing in PBS containing 0.1\% Triton X-100, the samples were sequentially labeled with TRITC-conjugated appropriate secondary antibodies (Zymed, San Francisco, CA) and the DNA stain 4'-6'-diamino-2-phenylindole (Molecular Probes, Eugene, OR). The coverslips were then mounted in VectaShield mounting medium (Vector Labs, Burlingame, CA) and sealed with nail polish. Samples were examined with a Zeiss Axiophot epifluorescence-equipped microscope.

\section{RESULTS}

Sperm samples from six patients were used in this study (Table 1). Although the original clinical diagnosis varied (Table 1), infertility in all samples was tentatively diagnosed as male factor using the recently described sperm-ubiquitin tag immunoassay (3).

In Patients 1 and 2 (Table 1), there were no obvious sperm morphological abnormalities (Fig. 1A, A'), and the predominant defect consisted of cells in the ejaculate as well as low motility in Patient 1 (Table 1). As expected, VAMP staining in these samples was identical to what had already been described in human sperm of proven fertility (1), with a strong label on the sperm head, prominent on the acrosome equatorial segment (Fig. 1A, arrows). Although some label was also found in the sperm tail, this staining is likely to be unspecific, since a similar pattern could also be obtained in control experiments, using only the fluorescent secondary antibodies, in the absence of any VAMP probe (data not shown).

Samples from patients 3 and 4 showed head and tail sperm defects, as well as low motility (Table 1). VAMP staining was frequently abnormal in these samples (Fig. 1B, B'). The most frequent departures from the norm, as assessed by VAMP staining, consisted of acrosomes that were not fully developed (Fig. 1B, arrows), as well as label in the neck/midpiece region of the sperm (Fig. 1B, arrowheads). These findings are most likely due to problems in acrosome formation during spermiogenesis and/or to abnormal processing of the residual cytoplasmic droplet. Some defects in sperm head condensation were also present (Fig. 1B', arrow).

In sperm of two patients with globozoospermia (patients 5 and 6, Table 1), VAMP staining was even more striking (Fig. 1C, C'). As noted previously (3), the sperm DNA is not fully condensed in these patients (Fig. 1C'). Sperm count and motility were low in patient 5 (Table 1), and sperm from patient 6 had large heads (Fig. 1C'). Furthermore, VAMP staining was only vestigial (Fig. 1C, arrows), which suggests that the acrosome had not fully formed. Indeed, the results obtained were reminiscent of what has been described re- 


\section{FIGURE 1}

VAMP in human sperm. (A), Normal VAMP staining in human sperm, with a concentration on the sperm head and the acrosome equatorial segment (arrows). Corresponding DNA image shown in A'. (B), Sperm with abnormal VAMP staining, which suggests misformed acrosomes (arrows), and abnormal presence of VAMP in the sperm midpiece (arrowheads). The DNA image (B') revealed misshapen heads in some cases (arrow). (C), Sperm from patients with globozoospermia, showing vestigial VAMP staining on the sperm head (arrows). For clarity, traces of the DNA staining (shown in C') have been superimposed on the VAMP image. Sperm from patient 5 is noted by an asterisk in C'. The bar represents 5 $\mu \mathrm{m}$.

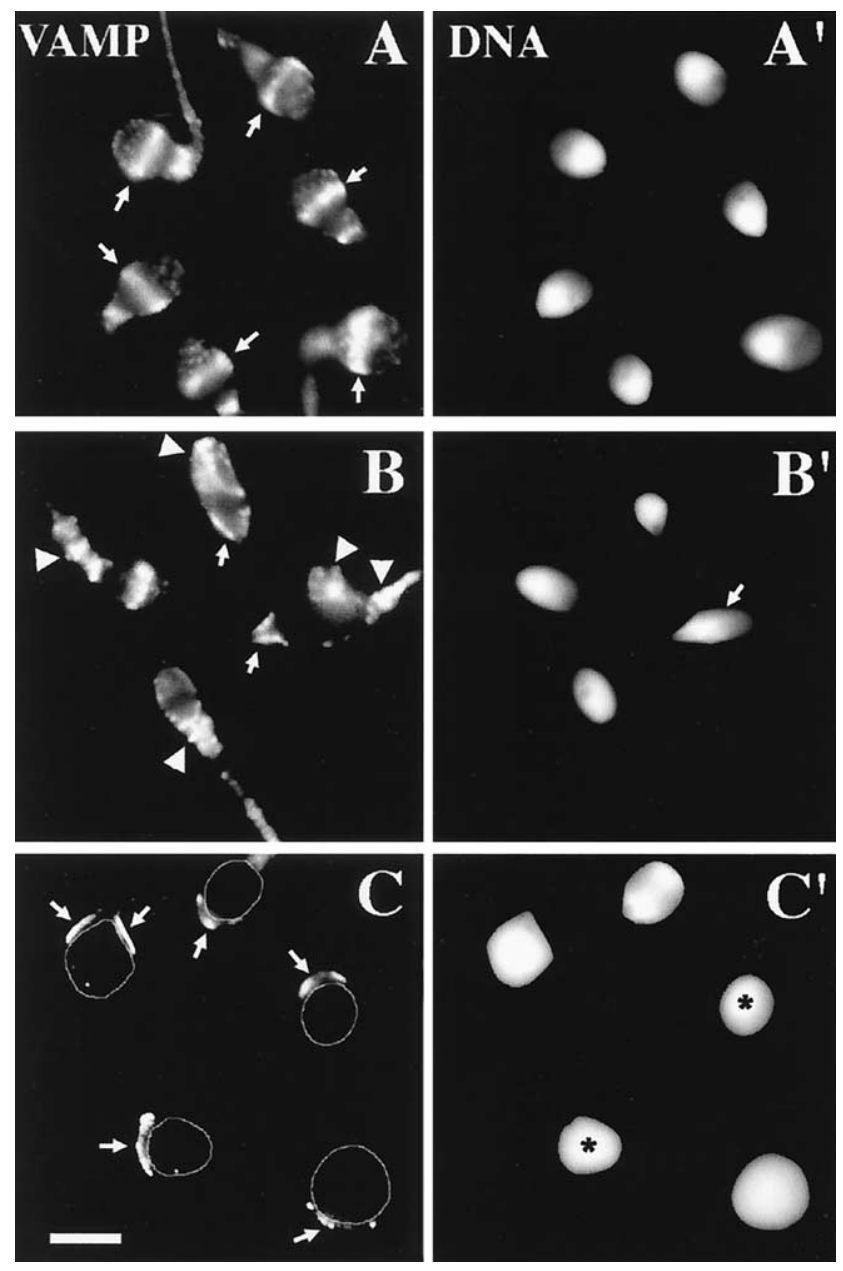

Ramalho-Santos. VAMP in human sperm. Fertil Steril 2002. garding the presence of VAMP in the developing acrosome of round spermatids (4).

\section{DISCUSSION}

The status and content of the human acrosome may be evaluated using several well-established techniques. In this study, we have used VAMP/synaptobrevin as a potential functional marker for the acrosome in the sperm of men participating in an infertility treatment program.

In some samples the acrosome seemed normal and poor sperm function could not be ascribed to organelle malformation or lack of membrane fusion modulators active during the acrosome reaction, such as VAMP. Clinically relevant parameters affected in these cases included low sperm motility or presence of cells in the ejaculate. However, in other instances there seemed to be some correlation between lower fertility and acrosome malformations as detected by VAMP staining. This was most evident in samples of patients with globozoospermia, where the acrosome was mostly vestigial, with similarities to the immature organelle found in round spermatids (4).

Since VAMP is involved in membrane fusion during the acrosome reaction (1), its presence, misplacement, or absence in the human acrosome may offer some indication as to the fertilization potential of human sperm.

Acknowledgments: The authors thank Dr. Gary Wessel, Brown University, for the kind gift of some of the VAMP antibodies used in this study. They also thank Dr. Teresa Almeida Santos (Faculty of Medicine, University of Coimbra) for helpful discussions and undying enthusiasm.

\section{References}

1. Ramalho-Santos J, Moreno RD, Sutovsky P, Chan AWS, Hewitson L, Wessel GM, Simerly CR, Schatten G. SNAREs in mammalian sperm: possible implications for fertilization. Dev Biol 2000;223:54-69.

2. Ramalho-Santos J, Sutovsky P, Simerly CR, Oko R, Wessel G, Hewitson L, Schatten G. ICSI choreography: fate of sperm structures after monospermic rhesus ICSI and first cell cycle implications. Hum Reprod 2000;15:2610-20.

3. Sutovsky P, Terada Y, Schatten G. Ubiquitin-based sperm assay for the diagnosis of male factor infertility. Hum Reprod 2001;16:250-258.

4. Ramalho-Santos J, Moreno RD, Wessel GM, Chan EKL, Schatten G. Membrane trafficking machinery components associated with the mammalian acrosome during spermiogenesis. Exp Cell Res 2001;267:45-60. 